Honam Mathematical J. 35 (2013), No. 4, pp. 679-681

http://dx.doi.org/10.5831/HMJ.2013.35.4.679

\title{
ON A QUESTION RELATED TO KOLMOGOROV FORWARD EQUATION
}

\author{
JOONGUL LEE
}

\begin{abstract}
In this paper, we raise a question regarding existence and uniqueness of the steady-state solution of a type of Kolmogorov forward equation. We prove that the question has a positive answer for trivial vector fields, and also provide examples which show that the compactness assumption is necessary.
\end{abstract}

\section{Introduction}

Let $\mathcal{M}$ be an orientable Riemannian manifold of dimension $n$, whose inner product at each point is denoted by $\langle$,$\rangle . The inner product gives$ us a canonical isomorphism between the 1 -forms on $\mathcal{M}$ and the vector fields on $\mathcal{M}$. For convenience we denote the isomorphism and its inverse by a tilde. Then if $f$ is a function on $\mathcal{M}$, its gradient is the vector field

$$
\nabla f=\tilde{d f} .
$$

And if $E$ is a vector field on $\mathcal{M}$, its divergence is the function

$$
\nabla \cdot E=* d * \widetilde{E}
$$

where $*$ is the Hodge star operator. (cf. [4])

Question. Assume $\mathcal{M}$ is compact. For a vector field $X$ on $\mathcal{M}$, is it possible to find unique vector fields $E, F$ with the following properties?

(1) $X=E+F$,

(2) $F=-\nabla \phi$ for some function $\phi$ on $\mathcal{M}$,

(3) $\langle E, F\rangle+\nabla \cdot E=0$ at every point of $\mathcal{M}$.

Received August 22, 2013. Accepted September 10, 2013.

2010 Mathematics Subject Classification. Primary 58J65; Secondary 53A45.

Key words and phrases. Kolmogorov forward equation, Fokker-Planck equation. This work was supported by 2011 Hongik University Research Fund. 


\section{Background}

Kolmogorov forward equation, also known as Fokker-Planck equation, describes the Brownian motion of particles. One may consult [3] for general background.

Given a vector field $X$ on $\mathbb{R}^{n}$, let us consider the following type of Kolmogorov forward equation

$$
\frac{\partial}{\partial t} \rho(x, t)=\nabla \cdot(-X+\nabla) \rho(x, t)
$$

where $\rho(x, t)$ is the probability density function at time $t$. We are interested in the steady-state solution of equation (1), i.e. $\frac{\partial \rho}{\partial t}=0$.

Let us assume that the steady-state solution is of the form

$$
\rho(x)=e^{-\phi(x)} .
$$

Let $F=-\nabla \phi$ and $E=X-F$. Then from the steady-state condition, we get

$$
\nabla \cdot\left(-e^{-\phi} X-e^{-\phi} \nabla \phi\right)=-\nabla \cdot\left(e^{-\phi} E\right)=0,
$$

which is equivalent to

$$
\langle E, F\rangle+\nabla \cdot E=0
$$

Conversely, if we can write $X=E+F$ satisfying equation (2) where $F$ is a conservative vector field (i.e. gradient of a function), then we obtain the steady-state solution of equation (1).

Note that we made choices of signs consistent with statistical physics. For more detailed discussion and application to statistical physics, one may consult [1], and [2].

\section{Main Result and Examples}

Theorem 1. If $X=0$, then the question has an affirmative answer for every compact $\mathcal{M}$.

Proof. $E=F=0$ is clearly a solution. For uniqueness, the equation becomes $\nabla \cdot E=\langle E, E\rangle$ in this case. By Stokes' theorem $0=\int_{\mathcal{M}} \nabla \cdot E=$ $\int_{\mathcal{M}}\langle E, E\rangle$ therefore $E=0$ (and hence $F=0$ ) on $\mathcal{M}$.

The following examples show that the solution of the question is in general not unique without the compactness assumption. 
Example 1. Let $\mathcal{M}=\mathbb{R}$ and $X=0$. Consider $\nabla \cdot E=\langle E, E\rangle$ which in this case becomes

$$
\frac{d y}{d x}=y^{2}
$$

We find that

$$
y=-\frac{1}{(x+c)}
$$

where $c$ is an arbitrary constant, is a solution of equation (3). Clearly every vector field on $\mathbb{R}$ is conservative, hence we have infinitely many solutions on an open subset of $\mathbb{R}$. Using the product of this solution, we have infinitely many solutions of the question when $\mathcal{M}$ is an open subset of $\mathbb{R}^{n}$ and $X=0$.

Example 2. Let $\mathcal{M}=\mathbb{R}^{n}$ where $n \geq 2$ and let $X$ be a nonzero constant vector field on $\mathcal{M}$. There are infinitely many ways to write a given vector as a sum of two vectors orthogonal to each other. Since a constant vector field is conservative (being the gradient of a linear function) and divergence-free, we conclude that there are infinitely many ways of writing $X=E+F$ satisfying the conditions of the question.

\section{References}

[1] Chulan Kwon and Ping Ao, Nonequilibrium steady state of a stochastic system driven by a nonlinear drift force, Physical Review E, 84(6):061106, 2011.

[2] Jae Dong Noh, Stochastic echo phenomena in nonequilibrium systems, arXiv:1212.2706, 12 (2012).

[3] H. Risken, The Fokker-Planck equation, volume 18 of Springer Series in Synergetics, Springer-Verlag, Berlin, second edition, 1989. Methods of solution and applications.

[4] Frank W. Warner, Foundations of differentiable manifolds and Lie groups, Scott, Foresman and Co., Glenview, Ill.-London, 1971.

Joongul Lee

Department of Mathematics Education, Hongik University,

Seoul 121-791, Korea.

E-mail: jglee@hongik.ac.kr 